\title{
Publication of NIH funded trials registered in ClinicalTrials.gov: cross sectional analysis
}

\author{
(c) $(1)(9)$ OPEN ACCESS
}

\author{
Joseph S Ross assistant professor of medicine ${ }^{12}$, Tony Tse program analyst at ClinicalTrials.gov ${ }^{3}$, \\ Deborah A Zarin director of ClinicalTrials.gov ${ }^{3}$, Hui Xu postgraduate house staff trainee ${ }^{4}$, Lei Zhou \\ postgraduate house staff traine ${ }^{4}$, Harlan M Krumholz Harold H Hines Jr professor of medicine and \\ professor of investigative medicine and of public health ${ }^{256}$
}

\begin{abstract}
${ }^{1}$ Section of General Internal Medicine, Department of Medicine, Yale University School of Medicine, New Haven, CT, USA; ${ }^{2}$ Center for Outcomes Research and Evaluation, Yale-New Haven Hospital, New Haven, CT; ${ }^{3}$ Lister Hill National Center for Biomedical Communications, National Library of Medicine, National Institutes of Health, Bethesda, MD, USA; ${ }^{4}$ Fuwai Hospital and Cardiovascular Institute, Chinese Academy of Medical Sciences and Peking Union Medical College, Beijing, China; ${ }^{5}$ Robert Wood Johnson Clinical Scholars Program and Section of Cardiovascular Medicine, Department of Medicine, Yale University School of Medicine, New Haven, CT; ${ }^{6}$ Section of Health Policy and Administration, Yale University School of Epidemiology and Public Health, New Haven, CT
\end{abstract}

\begin{abstract}
Objective To review patterns of publication of clinical trials funded by US National Institutes of Health (NIH) in peer reviewed biomedical journals indexed by Medline.

Design Cross sectional analysis.

Setting Clinical trials funded by NIH and registered within ClinicalTrials.gov (clinicaltrials.gov), a trial registry and results database maintained by the US National Library of Medicine, after 30 September 2005 and updated as having been completed by 31 December 2008, allowing at least 30 months for publication after completion of the trial.

Main outcome measures Publication and time to publication in the biomedical literature, as determined through Medline searches, the last of which was performed in June 2011.
\end{abstract}

Results Among 635 clinical trials completed by 31 December 2008, 294 $(46 \%)$ were published in a peer reviewed biomedical journal, indexed by Medline, within 30 months of trial completion. The median period of follow-up after trial completion was 51 months (25th-75th centiles 40-68 months), and 432 (68\%) were published overall. Among published trials, the median time to publication was 23 months (14-36 months). Trials completed in either 2007 or 2008 were more likely to be published within 30 months of study completion compared with trials completed before 2007 (54\% (196/366) v 36\% (98/269); $\mathrm{P}<0.001)$.

Conclusions Despite recent improvement in timely publication, fewer than half of trials funded by $\mathrm{NIH}$ are published in a peer reviewed biomedical journal indexed by Medline within 30 months of trial completion. Moreover, after a median of 51 months after trial completion, a third of trials remained unpublished.

\section{Introduction}

Today, there is an increasing emphasis on the successful translation of results from research into practice. This requires the timely dissemination of findings. While research results might be submitted directly to regulatory agencies, such as the Food and Drug Administration (FDA), physicians and policy makers generally depend on peer reviewed publications to learn about findings from clinical trials. Extensive research has shown, however, that the results of studies are often not shared publicly in a timely way and that between $25 \%$ and $50 \%$ of clinical trials remain unpublished even several years after completion, ${ }^{1-16}$ although this work was largely focused on industry funded studies. There are many possible reasons behind the delayed or non-publication of results from clinical trials, including lack of incentive to disseminate negative or unsupportive findings, time constraints, limited resources, changing interests, or even failure to have an article accepted by a journal.

Understanding the patterns of publication of research findings among publicly funded research, as opposed to industry funded research, is important because of the funding and the expectation for public benefit. Within the United States, the National Institutes of Health (NIH) is the leading and largest government agency responsible for biomedical and health related research and invests more than $\$ 12$ bn (about $£ 7600 \mathrm{~m}$ or $€ 8900 \mathrm{~m}$ ) of public resources in funding research in people or in clinical research, $\$ 3.5$ bn explicitly on clinical trials. ${ }^{17}$ These costs do not include the considerable contributions and costs incurred by the participants in the research. Previous work suggests that 
half to two thirds of all government funded studies were published two or more years after completion of the trial ${ }^{18} 19$ - that is, after completion of enrolment and observation - but these estimates included both US and other non-US government agencies. To our knowledge, the last study to focus on rates of publication solely among NIH funded trials was done nearly 20 years ago and examined trials funded by NIH in $1979 .{ }^{4}$

We examined patterns of publication in peer reviewed biomedical journals indexed by Medline among a sample of NIH funded trials registered within ClinicalTrials.gov (clinicaltrials.gov), a trial registry and results database maintained by the US National Library of Medicine. Although there is no mandate by NIH that all funded research must be registered in ClinicalTrials.gov, in 2005 the International Committee of Medical Journal Editors (ICMJE) began requiring registration as a prerequisite for publication in one of its member journals,${ }^{20}$ nearly always in ClinicalTrials.gov for US based studies, and the 2007 US FDA Amendments Act required sponsors of trials of FDA regulated products to register their trials at inception within ClinicalTrials.gov.

\section{Methods}

\section{Data source}

ClinicalTrials.gov is a publicly available trial registry and results database developed and maintained by the National Library of Medicine on behalf of the NIH. It represents the most comprehensive source for information about ongoing and completed publicly and privately funded trials within and outside the US. ClinicalTrials.gov uses a web based system to facilitate registration of clinical trials by the individual or organisation with primary responsibility for the study (such as the principal investigator or study sponsor). ${ }^{21}$ Multisite clinical trials using the same protocol are considered to be a single study in the registry. ClinicalTrials.gov includes both mandatory and optional data elements, and trials cannot be registered without adequate completion of all mandatory data elements (according to minimal quality review criteria), approval by a human participant review board (or equivalent), and conformity to the regulations of the appropriate national health authorities. Additional information about the registry is available from the National Library of Medicine. ${ }^{22}$

\section{Study sample}

Among more than 100000 studies registered within ClinicalTrials.gov on 10 November 2010, we limited our study sample to interventional studies or "clinical trials" (n=81 197; fig $1 \Downarrow$ ), defined by the National Library of Medicine as "studies in human beings in which individuals are assigned by an investigator based on a protocol to receive specific interventions. ${ }^{, 23} \mathrm{We}$ further limited our sample to trials registered on or after 13 September 2005 ( $n=62$ 428), the date by which any ongoing clinical trial, and after which all newly initiated trials, needed to be registered to be considered for publication in a journal that follows the ICMJE registration policy, ${ }^{20}$ as this requirement was associated with a large increase in trial registration within ClinicalTrials.gov. ${ }^{24}$ Many trials that had already begun enrolling patients, and some that were completed, were registered on or after this date to comply with the ICMJE policy. We then limited our sample to trials primarily or partially funded by the NIH by selecting registrations that listed at least one NIH institute or centre as the sponsor or collaborator $(n=6424)$. Finally, to provide a minimum of at least two years for investigators to prepare data for analysis, draft a manuscript, and complete the process required to publish their trial, we limited our sample to trials whose overall recruitment status had been updated to notify ClinicalTrials.gov that the trial was completed by 31 December $2008(n=635)$. A trial with a status of "completed" is defined by the National Library of Medicine as a trial that has ended and participants are no longer being examined or treated (that is, the last participant's last visit has occurred). ${ }^{23}$ We excluded NIH funded trials whose overall recruitment status was registered as completed but did not provide an actual study completion date because we would not be able to determine the time lapse between completion and publication for these trials.

\section{Publication status}

Two investigators (HX, LZ) independently determined the publication status of each trial in January 2011 using a search protocol applied in previous research. ${ }^{18}$ All searches began by first examining the "publication" field within ClinicalTrials.gov to determine if trial investigators provided a citation of an article that described trial results, as this field is used to display citations of trial results or other relevant research. This field is populated in two ways as either the sponsor or investigator can provide one or more entries or ClinicalTrials.gov will search Medline on a daily basis and extract all publications that have an indexed ClinicalTrials.gov identifier (NCT number). If no citation was provided we manually searched Medline with the NCT number. If we did not identify a publication, we searched Medline again with the intervention, condition studied, and name of the principal investigator (when provided in response to the "study official" field). The articles identified through the search were matched to the corresponding trial (when possible) using the following information from ClinicalTrials.gov: detailed description, location, enrolment, study start and completion dates, and primary and secondary outcome measures. Using the same search protocol a third investigator (JSR) independently determined the publication status in June 2011 for all trials that the previous two investigators had determined were unpublished.

Although Medline is not a complete repository of all publications in all biomedical journals, it remains the largest database of biomedical journal articles that can be searched freely with the National Library of Medicine PubMed system. As such, Medline is the source that nearly all physicians and policy makers depend on to learn about and obtain access to clinical trial findings.

\section{Time to publication}

For all trials for which a publication was identified in Medline, we determined the time to publication by calculating the duration of time (in months) that had lapsed between completion of the study and publication. The study completion date was determined from ClinicalTrials.gov, and the publication date was determined with Medline. For trials published online ahead of print, we used the date on which the publication was made available electronically as the publication date. For all trials for which a publication could not be identified, we determined the follow-up time by calculating the duration of time, in months, that had lapsed between completion of the study and our final search (June 2011).

\section{Study variables}

We obtained information on each trial reported by the study sponsor or investigator directly from the National Library of Medicine, including the following ClinicalTrials.gov data elements for each trial $^{23}$ : trial number, brief title, lead sponsor and collaborators, investigators and study official, study design, 
trial phase, intervention name, condition, population studied (age, sex, accepts healthy volunteers), target or actual enrolment, study start and completion dates, primary purpose, primary and secondary outcome measures, and publication citations. Investigators/sponsors self report data for their trials during initial registration and are expected to update the information as the trial progresses. We used the information provided in the trial classification data element to categorise study purpose as efficacy only, efficacy and safety, safety only, or indeterminate. For instance, a study design of "safety/efficacy study" was categorised as efficacy and safety, whereas a study design of "safety study" was categorised as safety only. We used the information provided in the study design data element to determine random allocation and comparator arms. We used the information provided in the trial primary purpose data element to categorise whether the trial was designed to evaluate an approach to treatment, prevention, or diagnosis or other type of intervention.

\section{Statistical analysis}

We conducted a descriptive analysis, estimating the publication rate among completed NIH funded trials registered within ClinicalTrials.gov overall and categorised by trial characteristics. Because our sample captured all completed NIH funded trials registered within ClinicalTrials.gov, we did not use inferential statistics to estimate associations among a larger population, with the exception being an examination of whether trials completed in 2007 or 2008 were published more quickly that those completed in earlier years. This analysis was prespecified and undertaken to evaluate whether or not the increased attention to research dissemination exemplified by enactment of the FDA Amendments Act had an impact on patterns of publication. Our hypothesis was that trials completed after 2007 would be published more quickly. In addition, we evaluated the time to publication using Kaplan-Meier analysis. Finally, among published and unpublished trials, we calculated the total targeted number of enrolled participants using summary statistics. Statistical analysis was performed with JMP 9.0 (SAS Institute, Cary, NC).

\section{Results}

\section{Trial characteristics}

We identified 635 completed NIH funded clinical trials for inclusion in our analysis. The National Institute of Mental Health $(n=76)$ and the National Institute of Allergy and Infectious Diseases ( $n=72$ ) funded the most trials in our sample (table $\Downarrow$ ). Among all trials, $66 \%(n=418)$ were of interventions for treatment and $26 \%(n=165)$ were trials of interventions for prevention or diagnostic tests; $45 \%(\mathrm{n}=278)$ enrolled (actual or target reported) 100 participants or more. Finally, 58\% $(n=366)$ were completed during either 2007 or 2008 , whereas $42 \%$ $(n=269)$ of trials were completed before 2007.

\section{Time to publication}

For all trials in our sample, at least 30 months elapsed between completion and our search for a publication in Medline describing the trial's findings. Publication rates in peer reviewed biomedical journals indexed by Medline increased as more time elapsed after trial completion, as $6 \%$ of trials were published within six months of study completion, $15 \%(\mathrm{n}=98)$ within 12 months, 35\% ( $\mathrm{n}=223)$ within 24 months, and 46\% ( $\mathrm{n}=294)$ within 30 months.
As secondary analyses, we examined time to trial publication after additional periods of time (fig $2 \Downarrow)$. For about $70 \%(n=444)$ of the trials in our sample, at least 42 months had elapsed between trial completion and our search for a publication describing the trial's findings. Among these, 54\% $(\mathrm{n}=241)$ of trials were published. Similarly, for about $44 \%(n=278)$ of the trials in our sample, at least 54 months had elapsed between trial completion and our search for a publication. Among these, $62 \%(n=173)$ were published.

\section{Overall trial publication}

Among all trials, the median period of follow-up after study completion was 51 months, (25th-75th centiles 40-68 months). Overall, $68 \%(n=432)$ of trials were published in peer reviewed biomedical journals indexed by Medline and 32\% ( $n=203)$ remained unpublished. Among published trials, the median time to publication was 23 months (14-36 months). Unpublished trials had a cumulative target (or actual) enrolment of about 60 000 participants.

\section{Trial characteristics and publication within $\mathbf{3 0}$ months of study completion}

Few trials had a publication rate within 30 months in excess of $60 \%$ (table $\downarrow$ ). Among treatment trials, $60 \%$ of trials registered as either a phase II/III or phase III study and $61 \%$ of trials conducted under an FDA Investigational New Drug Application (IND) or Investigational Device Exemption (IDE) were published within 30 months of study completion. Similarly, few trials had a publication rate within 30 months below $40 \%$.

Notably, $40 \%$ of single arm treatment trials and $39 \%$ and $36 \%$ of trials funded by the National Institute on Aging and National Institute of Mental Health, respectively, were published within 30 months of study completion. Trials completed in either 2007 or 2008 were more likely to be published within 30 months of study completion when compared with trials completed before 2007 (54\% (196/366) v 36\% (98/269), respectively, $\mathrm{P}<0.001$; fig $3 \Downarrow)$.

\section{Discussion}

In a sample of NIH funded clinical trials registered in ClinicalTrials.gov, we found that fewer than half of trials were published in a peer reviewed biomedical journal indexed in Medline within 30 months of trial completion, although there were more recent improvements in timely publication. Furthermore, after a median of 51 months after study completion, we found that about a third of NIH funded trials remained unpublished. Twenty years ago, a single study focused on trials funded by NIH in 1979 found that $93 \%$ of completed trials were published within 10 years, using a broader definition of publication that included research abstracts, book chapters, and other non-Medline indexed sources. ${ }^{4}$ In contrast, a larger body of work focused on industry sponsored studies suggested that between $25 \%$ and $50 \%$ of clinical trials remain unpublished even several years after completion. ${ }^{1-16}$ Our results suggest that patterns of publication are similar for publicly funded and for industry funded clinical trial research and that substantial amounts of publicly funded research data are not published and available to inform future research and practice.

The current culture of research needs to prioritise the timely public dissemination of research findings via peer reviewed journals among research funded by both public and private sources. Sponsors and investigators need to prioritise the publication process, managing it as closely and rigorously as any other part of the scientific process, regardless of the 
outcome: positive, negative, or inconclusive. In previous years, publishing negative findings from clinical trials was considered far more difficult, and trials with positive findings were more likely to be recommended for publication. ${ }^{26}$ Two factors, however, facilitate the publication of negative findings. Firstly, the growth in online only journals, such as those offered by the Public Library of Science (PLoS) and BioMed Central (BMC), and the practice of print journals publishing articles "online only" has reduced competition for space among print journals. Secondly, the ICMJE, among its uniform requirements for manuscripts submitted to biomedical journals, has issued a statement compelling the publication of trials with negative results. ${ }^{27}$

Clearly, many steps occur between the completion of data collection and the publication of the central research findings from a clinical trial, only some of which are under the control of the investigators. Preparations for publication and dissemination, however, can be made in advance of the trial's completion, including data management and cleaning, analysis planning, and drafting of parts of the manuscript. Among those $\mathrm{NIH}$ funded trials that were published in a peer reviewed biomedical journal indexed by Medline, some required as little as a few months for publication, others as long as five years. Trials that required several years to be published could potentially have been moved forward more expeditiously. Clear and reasonable expectations for timely dissemination are needed; perhaps the goal should be to have results from clinical research reported publicly 12-24 months after study completion.

Interestingly, in our sample, trials completed in 2007 or 2008 were published more quickly after study completion than trials completed before 2007, suggesting that the timeliness of dissemination could be improving. This issue deserves re-examination in future years.

\section{Implications}

To this end, US policies have been recently enacted that might further improve public reporting of results. Enacted in 2007, the FDA Amendments Act requires the sponsors or designated principal investigators of nearly all non-phase I trials of FDA regulated drugs, biologicals, and medical devices to register their trials at inception within ClinicalTrials.gov. Critically, this law also requires public reporting of summary results among trials of FDA approved drugs and devices within a legally mandated timeframe, often within 12 months of trial completion, regardless of publication status. Specifically, investigators must report results for all primary and secondary outcomes to the ClinicalTrials.gov results database for public posting. ${ }^{28}$ Importantly, the ICMJE has clarified that member journals should not consider reported results to be a previous publication if they were posted in ClinicalTrials.gov. ${ }^{29}$

Although the act should make findings from NIH funded research more available, there are many NIH funded trials that are not covered by it, such as trials of behavioural interventions and surgical procedures. Results from these trials can be voluntarily submitted to ClinicalTrials.gov, but there is no law or policy requiring reporting or publication of results from these studies. Since April 2008, federal law requires that any published article that describes the results of research funded wholly or in part by the NIH is to be made publicly available no more than one year after the date of publication through PubMed Central, as part of the NIH's public access programme. ${ }^{30}$ No policies exist, however, to ensure that the public has access to results from NIH funded research that is not published, although there are policies promoting data sharing among NIH projects receiving more than $\$ 500000$ in direct costs per year. ${ }^{31}$
Lack of dissemination of research findings disrupts the scientific process and leads to redundant efforts and misconceptions about clinical evidence, undermining not only the trial in question but also the evidence available in the medical literature that forms the basis for systematic reviews and meta-analyses, evidence based clinical and policy decisions, and even institutional review board assessments of risks and benefits associated with future research studies. In addition, non-publication violates the commitment made to trial participants, all of whom took part in the research and possibly placed themselves at some risk in an effort to contribute to clinical science. International guidelines on protecting participants in research, such as the Declaration of Helsinki, also recognise the ethical obligation in publishing or otherwise making publicly available "negative and inconclusive as well as positive results." 32 There are many studies "competing" for trial volunteers, yet we found that data from about $30 \%$ of participants in trials have not contributed to the publicly available knowledge base available through Medline 30 or more months after study completion. Given the concerns raised about insufficient numbers of volunteers for clinical trial research, ${ }^{33}$ a key step should be to ensure that data from all those who volunteer are published and widely used to inform future research and practice.

ClinicalTrials.gov and its results database offer a unique opportunity and venue for sponsors and investigators to report results from NIH funded clinical trials, as well as all other studies, to the broader public, regardless of whether or not the results are published in a peer reviewed journal. As a central repository already being used for trial registration, ${ }^{24}$ the site will be familiar to researchers and regulators. Although the legal requirements pertain only to certain interventional studies of drugs and devices, all studies registered in ClinicalTrials.gov are eligible to use the database for reporting summary results. Finally, using the results database ensures that summary data from trials are reported with tabular data forms that can be easily searched and do not limit reporting only to specific results relevant only to particular target audiences.

\section{Limitations}

The central limitation to our study is that we focused our search for relevant publications from NIH funded research to peer reviewed biomedical journals indexed by Medline and did not search other databases, such as Embase or research conference proceedings (abstracts). Our search for publications was extensive, however, involving two independent investigators using systematic methods. In addition, some trials might have been made publicly available elsewhere, such as through investigator or institutional websites. Another factor to consider is that even though Medline initiated efforts to index publications using ClinicalTrials.gov NCT numbers in July 2005, the practice didn't become common until later, perhaps making it more difficult to identify publications from older trials.

There are additional limitations to consider. This study relied on data in ClinicalTrials.gov that are reported by the trial sponsor or responsible investigator. The National Library of Medicine cannot verify the validity of the trial information, and there might be errors in some records. In addition, there is some uncertainty in our estimates of trial enrolment as for some trials within ClinicalTrials.gov only the target enrolment number was reported, which might differ from the actual enrolment number. Moreover, our study was limited to NIH funded trials and cannot be generalised to other international government agencies that fund clinical trial research. 
Finally, there are several reasons why our overall observation that $32 \%$ of NIH funded trials were not published in a peer reviewed biomedical journal indexed by Medline is a conservative estimate of non-publication. Firstly, we limited our analysis to trials that were registered and had updated their status to completed, indicating that they were no longer recruiting or observing participants. We would expect that some proportion of initiated trials would be registered and have difficulty with recruitment or follow-up but continue to pursue these goals without formally terminating or completing the trial. Secondly, we limited our analysis to trials that were registered and provided a study completion date. Our previous work has shown that completed trials that do not provide study completion dates within ClinicalTrials.gov were less likely to be published than those providing the information..$^{18}$ In addition, some institutes, such as the National Cancer Institute, were less likely to provide trial completion dates and were thus under-represented in our sample. Finally, we limited our analysis to trials that were completed by December 2008, providing a minimum of two and a half years for investigators to publish the trial findings in a peer reviewed biomedical journal indexed by Medline. We expect lower publication rates among trials that have had fewer than two years to publish the findings.

\section{Conclusion}

In conclusion, despite recent improvement in timely publication, overall we found that fewer than half of NIH funded trials in our sample registered within ClinicalTrials.gov were published in a peer reviewed biomedical journal indexed by Medline within 30 months of trial completion. Moreover, after a median of 51 months after trial completion, we found that a third of trials remained unpublished. Although there might be many reasons for lack of publication, the results database at ClinicalTrials.gov offers a complementary method of providing timely public access to study results, although the peer reviewed literature is likely to remain the principal method of communicating with clinicians and policy makers. Steps must be taken to ensure the timely dissemination of publicly funded research so that data from all those who volunteer are available to inform future research and practice.

Contributors: JSR, TT, DAZ, and HMK were responsible for the conception and design of this work. JSR, TT, HX, and LZ were responsible for acquisition of data. JSR drafted the manuscript, conducted the statistical analysis, and is guarantor. HMK obtained funding and provided supervision. All authors participated in the analysis and interpretation of the data and critically revised the manuscript for important intellectual content.

Funding: The analyses on which this publication is based were performed under Contract No. HHSN276201000788P, entitled "Evaluating Time-to-Publication Following Completion of Clinical Trials and Related Factors," funded by the US National Library of Medicine, National Institutes of Health, Department of Health and Human Services. The ideas and opinions expressed are those of the authors. The content of this publication does not necessarily reflect the views or policies of the US National Institutes of Health, Public Health Service, or Department of Health and Human Services. The authors assume full responsibility for the accuracy and completeness of the ideas presented. JSR and HMK receive support from Medtronic and from the Centers of Medicare and Medicaid Services (CMS) to develop and maintain performance measures that are used for public reporting. JSR is supported by the National Institute on Aging (K08 AG032886) and by the American Federation for Aging Research through the Paul B Beeson Career Development Award Program. DAZ and TT are supported by the Intramural Research Program of the National Institutes of Health,
National Library of Medicine. HMK is supported by a National Heart, Lung, and Blood Institute Cardiovascular Outcomes Center Award (1U01HL105270-02).

Competing interests: All authors have completed the ICMJE uniform disclosure form at www.icmje.org/coi_disclosure.pdf (available on request from the corresponding author) and declare: JSR is a member of a scientific advisory board for FAIR Health; HMK chairs a scientific advisory board for UnitedHealthcare; DAZ and TT are director and programme analyst, respectively, for ClinicalTrials.gov; no other relationships or activities that could appear to have influenced the submitted work.

Ethical approval: Not required.

Data sharing: Requests for statistical code and dataset can be made to the corresponding author.

Chan AW, Hrobjartsson A, Haahr MT, Gotzsche PC, Altman DG. Empirical evidence for selective reporting of outcomes in randomized trials: comparison of protocols to published articles. JAMA 2004;291:2457-65.

2 Chan AW, Krleza-Jeric K, Schmid I, Altman DG. Outcome reporting bias in randomized trials funded by the Canadian Institutes of Health Research. CMAJ 2004;171:735-40.

3 Decullier E, Lheritier V, Chapuis F. Fate of biomedical research protocols and publication bias in France: retrospective cohort study. BMJ 2005;331:19.

4 Dickersin K, Min YI. NIH clinical trials and publication bias. Online J Curr Clin Trials 1993;Apr 28:doc50

5 Dickersin K, Min YI, Meinert CL. Factors influencing publication of research results. Follow-up of applications submitted to two institutional review boards. JAMA 1992;267:374-8.

6 Easterbrook PJ, Berlin JA, Gopalan R, Matthews DR. Publication bias in clinical research. Lancet 1991;337:867-72.

7 loannidis JP. Effect of the statistical significance of results on the time to completion and publication of randomized efficacy trials. JAMA 1998;279:281-6.

8 Krzyzanowska MK, Pintilie M, Tannock IF. Factors associated with failure to publish large randomized trials presented at an oncology meeting. JAMA 2003;290:495-501.

9 Misakian AL, Bero LA. Publication bias and research on passive smoking: comparison of published and unpublished studies. JAMA 1998;280:250-3.

10 Stern JM, Simes RJ. Publication bias: evidence of delayed publication in a cohort study of clinical research projects. BMJ 1997;315:640-5.

11 Von Elm E, Rollin A, Blumle A, Huwiler K, Witschi M, Egger M. Publication and non-publication of clinical trials: longitudinal study of applications submitted to a research ethics committee. Swiss Med Wkly 2008:138:197-203.

12 Turner EH, Matthews AM, Linardatos E, Tell RA, Rosenthal R. Selective publication of antidepressant trials and its influence on apparent efficacy. N Engl J Med 2008;358:252-60.

13 Bardy AH. Bias in reporting clinical trials. Br J Clin Pharmacol 1998;46:147-50.

14 Dwan K, Altman DG, Arnaiz JA, Bloom J, Chan AW, Cronin E, et al. Systematic review of the empirical evidence of study publication bias and outcome reporting bias. PLOS ONE 2008;3:e3081.

15 Lee K, Bacchetti P, Sim I. Publication of clinical trials supporting successful new drug applications: a literature analysis. PLoS Med 2008;5:e191.

16 Rising K, Bacchetti P, Bero LA. Reporting bias in drug trials submitted to the Food and Drug Administration: review of publication and presentation. PLoS Med 2008;5:e217.

17 US Department of Health and Human Services, National Institutes of Health. Research portfolio online reporting tools (RePORT): estimates of funding for various research, condition, and disease categories. 2011. http://report.nih.gov/rcdc/categories/.

18 Ross JS, Mulvey GK, Hines EM, Nissen SE, Krumholz HM. Trial publication after registration in ClinicalTrials.gov: a cross-sectional analysis. PLoS Med 2009;6:e1000144.

19 Bourgeois FT, Murthy S, Mandl KD. Outcome reporting among drug trials registered in ClinicalTrials.gov. Ann Intern Med 2010;153:158-66.

20 DeAngelis CD, Drazen JM, Frizelle FA, Haug C, Hoey J, Horton R, et al. Clinical trial registration: a statement from the International Committee of Medical Journal Editors. JAMA 2004;292:1363-4.

21 US National Institutes of Health. About ClinicalTrials.gov. www.clinicaltrials.gov/ct2/info/ about.

22 US National Institutes of Health. ClinicalTrials.gov. www.clinicaltrials.gov/.

23 US National Institutes of Health. ClinicalTrials.gov protocol data element definitions. http: //prsinfo.clinicaltrials.gov/definitions.html.

24 Zarin DA, Tse T, Ide NC. Trial registration at ClinicalTrials.gov between May and October 2005. N Engl J Med 2005;353:2779-87.

25 US National Institutes of Health. Glossary of clinical trial terms. www.clinicaltrials.gov/ct2/ info/glossary.

26 Emerson GB, Warme WJ, Wolf FM, Heckman JD, Brand RA, Leopold SS. Testing for the presence of positive-outcome bias in peer review: a randomized controlled trial. Arch Intern Med 2010;170:1934-9.

27 International Committee of Medical Journal Editors. Uniform requirements for manuscripts submitted to biomedical journals: publishing and editorial issues related to publication in biomedical journals: obligation to publish negative studies. www.icmje.org/publishing 1negative.html.

28 Zarin DA, Tse T, Williams RJ, Califf RM, Ide NC. The ClinicalTrials.gov results database-update and key issues. N Engl J Med 2011;364:852-60.

29 International Committee of Medical Journal Editors. Uniform requirements for manuscripts submitted to biomedical journals: publishing and editorial issues related to publication in biomedical journals: obligation to register clinical trials. www.icmje.org/publishing 10register.html.

30 US Department of Health and Human Services, National Institutes of Health, Public Access. NIH public access policy details. 2009. http://publicaccess.nih.gov/policy.htm.

31 US Department of Health and Human Services, National Institutes of Health, Office of Extramural Research. NIH data sharing policy. 2007. http://grants.nih.gov/grants/policy/ data_sharing/. 


\section{What is already known on this topic}

Previous work, largely focused on industry sponsored studies, suggests that between $25 \%$ and $50 \%$ of clinical trials remain unpublished even several years after completion

Little is known about the patterns of publication of publicly funded trials

\section{What this study adds}

Fewer than half of NIH funded trials registered after September 2005 within ClinicalTrials.gov and completed by December 2008 were published in a peer reviewed biomedical journal indexed by Medline within 30 months of trial completion

After a median of 51 months after study completion, a third of $\mathrm{NIH}$ funded trials in our sample remained unpublished

32 World Medical Association. Declaration of Helsinki-ethical principles for medical research involving human subjects. Adopted by the 59th WMA General Assembly, Seoul, Korea, October 2008. www.wma.net/en/30publications/10policies/b3/index.html.

33 Giffin RB, Woodcock J. Comparative effectiveness research: who will do the studies? Health Aff (Millwood) 2010;29:2075-81.

34 Krall RL. State of the controlled clinical trial enterprise in the United States. Clin Pharmacol Ther 2011;89:225-8.

Accepted: 01 November 2011

\section{Cite this as: BMJ 2012;344:d7292}

This is an open-access article distributed under the terms of the Creative Commons Attribution Non-commercial License, which permits use, distribution, and reproduction in any medium, provided the original work is properly cited, the use is non commercial and is otherwise in compliance with the license. See: http://creativecommons.org/licenses/bync/2.0/ and http://creativecommons.org/licenses/by-nc/2.0/legalcode. 


\section{Table}

Table 1 | Characteristics of completed NIH funded trials registered within ClinicalTrials.gov* and publication in peer reviewed biomedical journal indexed by Medline within $\mathbf{3 0}$ months of study completion, stratified by trial characteristics

\begin{tabular}{|c|c|c|}
\hline & No $(\%)(n=635)$ & No (\%) published \\
\hline \multicolumn{3}{|l|}{ NIH funding institute†: } \\
\hline National Cancer Institute (NCl) & $49(8)$ & $25(51)$ \\
\hline National Heart, Lung, and Blood Institute (NHLBI) & $54(9)$ & $23(43)$ \\
\hline $\begin{array}{l}\text { National Institute of Child Health and Human Development } \\
\text { (NICHD) }\end{array}$ & $53(8)$ & $23(43)$ \\
\hline National Institute on Aging (NIA) & $28(4)$ & $11(39)$ \\
\hline National Institute of Allergy and Infectious Diseases (NIAID) & $72(11)$ & $36(50)$ \\
\hline $\begin{array}{l}\text { National Institute of Diabetes and Digestive and Kidney Diseases } \\
\text { (NIDDK) }\end{array}$ & $55(9)$ & $30(55)$ \\
\hline National Institute of Mental Health (NIMH) & $76(12)$ & $27(36)$ \\
\hline $\begin{array}{l}\text { National Institute on Alcohol Abuse and Alcoholism } \\
\text { (NIAAA)/National Institute on Drug Abuse (NIDA) }\end{array}$ & $87(14)$ & $44(51)$ \\
\hline Other institute or not specified & $161(25)$ & $75(47)$ \\
\hline \multicolumn{3}{|l|}{ Focus: } \\
\hline Treatment & $418(66)$ & $206(49)$ \\
\hline Prevention or diagnostic & $165(26)$ & $70(42)$ \\
\hline Other (such as health services research or basic science) & $52(8)$ & $18(35)$ \\
\hline \multicolumn{3}{|l|}{ Purposeł: } \\
\hline Safety and efficacy & $149(36)$ & $71(48)$ \\
\hline Efficacy only & $224(54)$ & $115(51)$ \\
\hline Safety only & $20(5)$ & $11(55)$ \\
\hline Pharmacokinetics & $25(6)$ & $9(36)$ \\
\hline \multicolumn{3}{|l|}{ Conducted under FDA IND or IDE‡: } \\
\hline Yes & $79(22)$ & $48(61)$ \\
\hline No & $282(78)$ & $140(50)$ \\
\hline \multicolumn{3}{|l|}{ Phaseł: } \\
\hline I & $61(15)$ & $27(44)$ \\
\hline I/II or II & $137(33)$ & $65(47)$ \\
\hline IIIIII or III & $53(13)$ & $32(60)$ \\
\hline IV & $36(9)$ & $18(50)$ \\
\hline No phase designated & $131(31)$ & $64(49)$ \\
\hline \multicolumn{3}{|l|}{ Design, treatment groups§: } \\
\hline Single arm trial & $113(19)$ & $45(40)$ \\
\hline Multiple arm trial & $470(81)$ & $231(49)$ \\
\hline \multicolumn{3}{|l|}{ Design, control group among multiple arm studies§: } \\
\hline Placebo control group & $219(47)$ & $105(48)$ \\
\hline At least one active control group & $251(53)$ & $126(50)$ \\
\hline \multicolumn{3}{|l|}{ Design, group assignment among multiple arm studies§: } \\
\hline Randomisation used & $451(96)$ & $221(49)$ \\
\hline Randomisation not used & $19(4)$ & $10(53)$ \\
\hline \multicolumn{3}{|l|}{ Data monitoring board§: } \\
\hline Yes & $176(56)$ & $93(53)$ \\
\hline No & $140(44)$ & $64(46)$ \\
\hline \multicolumn{3}{|l|}{ Population studied included children: } \\
\hline Yes & $153(24)$ & $68(44)$ \\
\hline No & $482(76)$ & $226(47)$ \\
\hline
\end{tabular}


(continued)

\begin{tabular}{lcc} 
& No $(\%)(\mathbf{n}=635)$ & No (\%) published \\
\hline Population studied included older adults (age $\geq 65):$ & $331(52)$ & $153(46)$ \\
\hline Yes & $304(48)$ & $141(46)$ \\
\hline No & & $149(45)$ \\
\hline Trial enrolment (target or actual): & $334(55)$ & $133(48)$ \\
\hline$<100$ participants & $278(45)$ & $241(46)$ \\
\hline$\geq 100$ participants & & $25(56)$ \\
\hline Trial location includes at least one US enrolment site: & $524(92)$ & $98(36)$ \\
\hline Yes & $45(8)$ & $196(54)$ \\
\hline No & & $11(42)$ \\
\hline Trial completion date: & $269(42)$ & $283(47)$ \\
\hline Before 2007 & $366(58)$ & \\
\hline During 2007-8 & & $26(4)$ \\
\hline Trial results posted on ClinicalTrials.gov: & $609(96)$ & \\
\hline Yes & & \\
\hline No & & \\
\hline
\end{tabular}

$\mathrm{NIH}=$ National Institutes of Health; FDA=Food and Drug Administration; IND=Investigational New Drug Application; IDE=Investigational Device Exemption. ${ }^{*}$ All data elements not provided for each registered trial.

†Clinical trials cofunded by multiple NIH institutes assigned to institute listed first in ClinicalTrials.gov registration.

$\ddagger$ Data provided only for treatment clinical trials.

$\S$ Data provided only for treatment, prevention, and diagnostic clinical trials. 


\section{Figures}

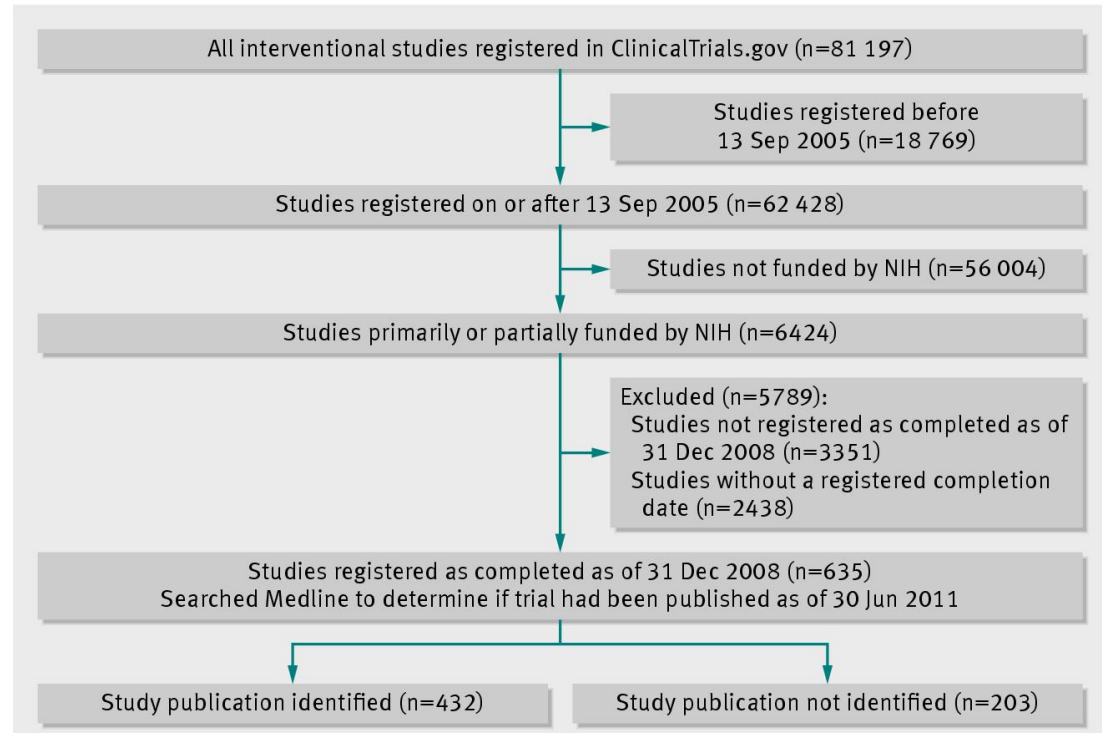

Fig 1 Inclusion of NIH funded trials registered within ClinicalTrials.gov

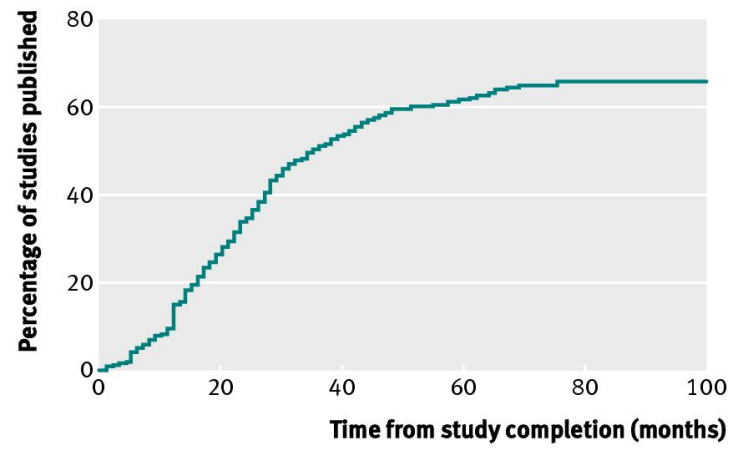

No at risk

$\begin{array}{lllllllllll}635 & 635 & 635 & 635 & 493 & 330 & 220 & 153 & 95 & 54 & 44\end{array}$

Fig 2 Cumulative percentage of studies published in a peer reviewed biomedical journal indexed by Medline during 100 months after trial completion among all NIH funded clinical trials registered within ClinicalTrials.gov 


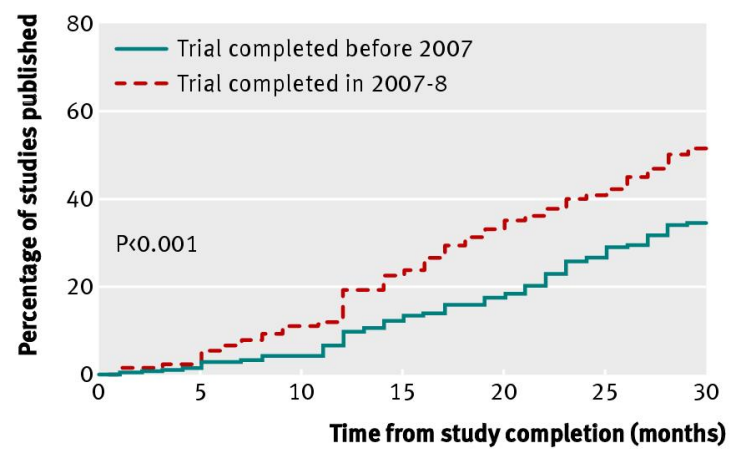

No of unpublished studies

Trial completed before 2007

$$
\begin{array}{lllllll}
269 & 264 & 259 & 235 & 221 & 197 & 175
\end{array}
$$

Trial completed in 2007-8

$\begin{array}{lllllll}366 & 356 & 324 & 282 & 244 & 215 & 176\end{array}$

Fig 3 Cumulative percentage of studies published in peer reviewed biomedical journal indexed by Medline during 30 months after trial completion among $\mathrm{NIH}$ funded clinical trials registered within ClinicalTrials.gov, stratified by whether trial was completed before 2007 or during 2007-8 